\title{
Event-Related Potentials in Parkinson's Disease Patients with Visual Hallucination
}

\author{
Yang-Pei Chang, ${ }^{1}$ Yuan-Han Yang, ${ }^{1,2}$ Chiou-Lian Lai, ${ }^{2,3}$ and Li-Min Liou ${ }^{2,3}$ \\ ${ }^{1}$ Department of Neurology, Kaohsiung Municipal Ta-Tung Hospital, Kaohsiung Medical University, Kaohsiung, Taiwan \\ ${ }^{2}$ Department of Neurology, Faculty of Medicine, College of Medicine, Kaohsiung Medical University, Kaohsiung, Taiwan \\ ${ }^{3}$ Department of Neurology, Kaohsiung Medical University Hospital, Kaohsiung, Taiwan \\ Correspondence should be addressed to Chiou-Lian Lai; cllai@kmu.edu.tw
}

Received 22 September 2016; Accepted 9 November 2016

Academic Editor: Jan Aasly

Copyright (c) 2016 Yang-Pei Chang et al. This is an open access article distributed under the Creative Commons Attribution License, which permits unrestricted use, distribution, and reproduction in any medium, provided the original work is properly cited.

\begin{abstract}
Using neuropsychological investigation and visual event-related potentials (ERPs), we aimed to compare the ERPs and cognitive function of nondemented Parkinson's disease (PD) patients with and without visual hallucinations (VHs) and of control subjects. We recruited $12 \mathrm{PD}$ patients with VHs (PD-H), 23 PD patients without VHs (PD-NH), and 18 age-matched controls. All subjects underwent comprehensive neuropsychological assessment and visual ERPs measurement. A visual odd-ball paradigm with two different fixed interstimulus intervals (ISI) $(1600 \mathrm{~ms}$ and $5000 \mathrm{~ms}$ ) elicited visual ERPs. The frontal test battery was used to assess attention, visual-spatial function, verbal fluency, memory, higher executive function, and motor programming. The PD-H patients had significant cognitive dysfunction in several domains, compared to the PD-NH patients and controls. The mean P3 latency with ISI of $1600 \mathrm{~ms}$ in PD-H patients was significantly longer than that in controls. Logistic regression disclosed UPDRS-on score and P3 latency as significant predictors of VH. Our findings suggest that nondemented PD-H patients have worse cognitive function and P3 measurements. The development of VHs in nondemented PD patients might be implicated in executive dysfunction with altered visual information processing.
\end{abstract}

\section{Introduction}

Visual hallucinations (VHs) and cognitive impairment, which are nonmotor symptoms of Parkinson's disease (PD), have been an intriguing issue in recent years [1]. It is crucial to screen mild cognitive impairment and dementia in PD patients because dementia may cause nursing home placement, increased burden for health care and caregiver, and higher mortality [2]. In the mid-stage of PD, VHs act as a clinical predictor of dementia $[3,4]$ and correlate with disease progression and decline in Mini-Mental State Examination (MMSE) scores [5, 6]. Recent hypotheses suggest that the development of VHs in PD may result from an imbalance of external and internal inputs and impaired reality monitoring, while cognitive impairment may play a role in reality monitoring $[7,8]$. Cognitive correlation of VHs in PD patients is evident [9-12]. A one-year neuropsychological follow-up study reported that nondemented PD patients with VHs have faster decline of complex visual function and multiple cognitive domains than patients without VHs [13]. Previous studies have also reported worse attention and visuospatial function in PD patients with VHs $[14,15]$. However, another 4-year longitudinal observatory study showed that VHs may be more associated with longer disease duration, increased functional impairment, and premorbid psychiatry illness rather than cognitive impairment [16]. Accumulating evidence has demonstrated that cognitive dysfunction may contribute to the occurrence of $\mathrm{VH}$ symptoms of PD patients in nondemented PD patients with VHs regardless of the side effect of dopaminergic medication [17, 18]. Indeed, recent study using functional MRI technique suggests desynchronization between aberrant frontal circuit and posterior cortical areas during active visual hallucinations [19].

Event-related potential (ERP) is a developed sensitive and noninvasive tool to detect cognitive dysfunction in patients with mild cognitive impairment and dementia [20-22]. Early components of ERP (N1 and P2) are considered exogenous sensory components that have been associated with attention 
and sensory processing. The N2 component reflects an early detection of cognitive ability, such as target discrimination. P3 is a positive shift when a subject detects an informative task-relevant stimulus $[23,24]$. While some studies have supported the correlation between ERP abnormality and cognitive impairment in PD patients with dementia, the role of ERP in nondemented PD patients is not confirmed [25-31]. One study found visual cognitive impairment and prolonged visual P3 latency especially in patients with PD dementia with hallucinations [32].

Since ERP may be a sensitive tool in the detection of cognitive impairment in $\mathrm{PD}$ in the absence of clinical dementia and $\mathrm{VH}$ is a potentially premonitory symptom of dementia in PD patients, it may be interesting to explore the ERP abnormality in nondemented PD patient with VHs. In the literature, few studies focused on the topic. We aim to assess the visual ERP and neuropsychological assessments in nondemented $\mathrm{PD}$ patients with $(\mathrm{PD}-\mathrm{H})$ and without $\mathrm{VH}$ $(\mathrm{PD}-\mathrm{NH})$ and healthy controls and find the linkage.

\section{Materials and Methods}

2.1. Participants. This study was conducted at the Kaohsiung Medical University Hospital (KMUH), a tertiary referral center in Southern Taiwan. The KMUH institution review board approved all procedures and written informed consent was obtained from study participants. The control subjects were recruited from volunteer in nearby community college. All PD participants had a presumptive clinical diagnosis of PD according to UKPD Brain Bank criteria. Individuals were inquired carefully and were assigned to groups according to whether they had experienced VHs in the past one year. No patient in the population sampled had a clinical diagnosis of either Alzheimer's disease or Lewy body dementia. Patients were excluded if Mini-Mental State Examination (MMSE) is less than 25. Patients with eye disease or migraine or other conditions like concurrent stroke, delirium, delusions, multiple sclerosis, and psychiatric illness or those under neuroleptics treatment were all excluded. Duration of illness and medication were recorded and stage of illness was scored according to the Hoehn and Yahr scale and United Parkinson's Disease Rating Scale (UPDRS) during “on” state. PD patients take neuropsychological assessment and eventrelated potential during "on" state after regular oral medications.

2.2. Neuropsychological Assessment. The neuropsychological assessment focusing on frontal lobe function [21] includes Mini-Mental State Examination (MMSE), Digit Span (Wechsler Adult Intelligence Scale-Revision (WAIS-R)), Digit Symbol (WAIS-R), Stroop Test, and Trail Making Tests (parts A and $\mathrm{B}$ ) to assess attention and concentration; Block Design (WAIS-R) and Rey-Osterrieth Complex Figure Test-Copy to evaluate visuoconstructional ability; word list generation (Controlled Oral Word Association; Category Fluency Test) to assess semantic verbal fluency; word list learning-recall and Rey-Osterrieth Complex Figure Test-Recall to evaluate memory; Wisconsin Card Sorting Test-Modified (WCST),
Design Fluency (Five-Point Test), and Similarity (WAIS-R) to assess higher executive function; Luria's Hand Sequence to evaluate motor programming function.

2.3. Event-Related Potentials Measurements. A visual "oddball” stimulus paradigm (NeuroStim, NeuroScan, Inc.) was used to elicit visual event-related potentials, and an electroencephalograph (EEG) was recorded using $\mathrm{Ag} / \mathrm{AgCl}$ electrodes placed at 5 scalp locations $(\mathrm{FPz}, \mathrm{Fz}, \mathrm{Cz}, \mathrm{Pz}$, and $\mathrm{Oz})$, based on the 10-20 system. All were referenced to linked earlobes. The electrode impedance was kept below $5 \mathrm{k} \Omega$. The EEG was amplified (band pass, $0.01-40 \mathrm{~Hz}$ ) by a SynAmps amplifier (NeuroScan, Inc.), and continuous EEG records were kept for further offline analysis at a sampling rate of $256 \mathrm{~Hz}$. The averaging epoch was $1024 \mathrm{~ms}$, including $200 \mathrm{~ms}$ of prestimulus baseline [21,22].

The subjects sat in a comfortable chair in a soundattenuated room with dim lighting $100 \mathrm{~cm}$ in front of a 19inch LCD computer screen. Stimuli were presented in the central of the screen. The stimuli consisted of two neutral pictures from the NeuroScan template on a dark ground. The participants were asked to centrally fixate throughout the recording. We adopted a visual odd-ball task, with a target stimulus and a nontarget stimulus. Mistrials including eyeball movement artifacts were excluded from the offline analysis.

Stimuli were presented randomly with the probability of $20 \%$ target stimulus and $80 \%$ nontarget stimuli. In each block a total of 250 stimuli (50 targets, 200 nontargets) were presented for $100 \mathrm{~ms}$ and interstimulus interval (ISI) of $1600 \mathrm{~ms}$ and $5000 \mathrm{~ms}$. The experiment consisted of 4 blocks ( 2 blocks with ISI of $1600 \mathrm{~ms}$ and 2 blocks with ISI of $5000 \mathrm{~ms}$ ). Participants performed a brief training session to ensure they were able to detect the target accurately. During the examination, participants were asked to press the button as quickly as possible when they saw the target. Reaction time was measured relative to target onset for correct trials, while accuracy was measured as the percentage of correct responses out of all responses to the target stimulus. Individual trials with eye blink artifacts (more than $250 \mu \mathrm{V}$ of peak-to-peak amplitude), target trials for which the reaction time (RT) was more than $1.4 \mathrm{~s}$, and nontarget trials with a response were all excluded from the averaging. Separate ERP averages were made for each trial type. For amplitudes analysis, the mean potential during the $200 \mathrm{~ms}$ period preceding the stimulus onset served as baseline. The N1, P2, $\mathrm{N} 2$, and $\mathrm{P} 3$ components at $\mathrm{Pz}$ recording were assessed for highest amplitude distribution. The latencies windows were N1 component as the maximum negativity between 75 and $160 \mathrm{~ms}, \mathrm{P} 2$ component as the maximum positivity between 170 and $260 \mathrm{~ms}$, N2 component as the maximum negativity between 190 and $360 \mathrm{~ms}$, and P3 component as the maximum positivity between 250 and $500 \mathrm{~ms}$.

2.4. Statistics. We performed statistical analysis with SPSS 12.0 package, and $p<0.05$ was set to be statistically significant. We used two-tailed $t$-test for analyzing continuous data of disease characteristic of PD patients. We used analysis of 
TABLE 1: Characteristics in PD-H, PD-NH, and controls.

\begin{tabular}{|c|c|c|c|c|}
\hline & $\begin{array}{l}\text { PD-H } \\
n=12\end{array}$ & $\begin{array}{c}\mathrm{PD}-\mathrm{NH} \\
n=23\end{array}$ & $\begin{array}{c}\text { Controls } \\
n=18\end{array}$ & $p$ \\
\hline Age $^{1}$, years & $67.79 \pm 7.93$ & $66.36 \pm 9.68$ & $68.29 \pm 6.83$ & 0.753 \\
\hline Women $^{1}, n(\%)$ & $5(36)$ & $7(28)$ & $10(59)$ & 0.107 \\
\hline Education $^{1}, \mathrm{y}$ & $9.27 \pm 6.65$ & $10.88 \pm 4.16$ & $12.16 \pm 3.32$ & 0.245 \\
\hline Disease duration $^{2}, \mathrm{y}$ & $11.73 \pm 6.41$ & $6.20 \pm 4.86$ & $\mathrm{n} / \mathrm{a}$ & 0.007 \\
\hline Duration of levodopa ${ }^{2}, y$ & $8.44 \pm 5.78$ & $3.04 \pm 3.57$ & $\mathrm{n} / \mathrm{a}$ & 0.004 \\
\hline $\mathrm{H} \& \mathrm{Y}^{2}$ & $2.65 \pm 0.89$ & $1.52 \pm 0.65$ & $\mathrm{n} / \mathrm{a}$ & $<0.001$ \\
\hline MMSE $^{1}$ (score) & $27.73 \pm 2.20$ & $27.58 \pm 1.36$ & $28.41 \pm 1.37$ & 0.472 \\
\hline $\mathrm{HDI}^{1}$ (score) & $5.00 \pm 4.67$ & $4.125 \pm 4.11^{*}$ & $0.25 \pm 0.79$ & $<0.001$ \\
\hline UPDRS-III motor ${ }^{2}$ (score) & $27.92 \pm 13.00$ & $14.20 \pm 8.42$ & $\mathrm{n} / \mathrm{a}$ & $<0.001$ \\
\hline Levodopa-equivalent dose & $863.8 \pm 390.6$ & $311.2 \pm 160.5$ & $\mathrm{n} / \mathrm{a}$ & 0.08 \\
\hline
\end{tabular}

PD-H: PD patients with visual hallucinations; PD-NH: PD patients without visual hallucinations; H\&Y: Hoehn and Yahr stage; HDI: Hamilton depression index; UPDRS: Unified Parkinson's Disease Rating Scale; n/a: not available. $p$ : $p$ value, $p<0.05$; by ${ }^{1}$ ANOVA $^{2} t$-test.

covariate (ANCOVA) to determine the differences of neuropsychological test between groups after adjusting disease duration, duration of levodopa use, Hoehn and Yahr stage, Hamilton depression index, and the scores of UPDRS-III. For P3 latency and amplitude, we used ANCOVA to determine the significant difference after adjusting age, gender, disease duration, duration of levodopa use, and Hoehn and Yahr stage with Tukey method used for post hoc analysis. Oneway repeated measures analysis of variance (ANOVA) was used to explore the effect of ISI on P3 latency and amplitude. Pearson correlation coefficient was calculated to explore the relationship between neuropsychological function and $\mathrm{N} 1$, P2, N2, and P3 latency and amplitude at Pz recording. ERP components at $\mathrm{Fz}$ and $\mathrm{Cz}$ recordings were not analyzed because of artifacts. For our intent in analyzing the predictive risk factors of $\mathrm{VH}$, logistic regression with the existence or absence of VHs as dependent variable was performed in four different models with different confounding factors. To clarify the role of UPDRS-on score in the development of VH, we adjusted age and gender in model 1 , while we adjusted age, gender, and three cognitive domains in model 3. To further observe the role of P3 latency in the development of $\mathrm{VH}$, age and gender and UPDRS-on score were adjusted in model 2, while age and gender, UPDRS-on score, and three cognitive domains were adjusted in model 4 . The chosen cognitive domains (Trail Making Tests, R-O copy, or Luria Hand Sequence) were significantly different between PD$\mathrm{H}$ and PD-NH patients. According to WAIS-III Chinese version, the cut-off values of three cognitive domains were chosen and were transformed to dichotomy dummy variable for logistic regression.

\section{Results}

3.1. Demographic Data. Twelve PD-H patients, twenty-three $\mathrm{PD}-\mathrm{NH}$ patients, and eighteen healthy control subjects were recruited in this study (Table 1). The mean age, education level, and MMSE did not differ significantly between these three groups, while there were significant differences between the PD patients with and without visual hallucinations with regard to disease duration, duration of levodopa use, Hoehn and Yahr stage, and the scores of UPDRS-III. We also found significant difference in Hamilton depression index in PD-H or PD-NH patients when comparing with normal controls.

3.2. Neuropsychological Assessment. The data of neuropsychological investigations in all participants are shown in Table 2. There were multiple domains of frontal dysfunction in PD patients, especially in PD-H patients. The PD-H patients performed significantly worse than normal controls at Trail Making Test, R-O copy, Wisconsin Card Sorting Test, and Luria Hand Sequence. Moreover, when comparing $\mathrm{PD}-\mathrm{H}$ patients to $\mathrm{PD}-\mathrm{NH}$ patients, significantly lower scores were found in the former group at Trail Making Test, RO copy, Wisconsin Card Sorting Test, and Luria's Hand Sequence. When comparing to normal controls, PD-NH patients performed significantly worse in Wisconsin Card Sorting Test.

3.3. Visual ERP Data. For the highest amplitude distribution, the N1, P2, N2, and P3 components with two different ISI at $\mathrm{Pz}$ are outlined in Table 3. There was no significant difference between PD-H patients, PD-NH patients, and controls, regardless of different ISI $(1600 \mathrm{~ms}$ and $5000 \mathrm{~ms})$. However, the mean latency of P3 with ISI of $1600 \mathrm{~ms}$ in PD$\mathrm{H}$ patients revealed significant prolongation when comparing with that in controls. The mean reaction time and error rate of $\mathrm{PD}-\mathrm{H}$ patients, $\mathrm{PD}-\mathrm{NH}$ patients, and controls revealed no significant difference.

We also assessed the effect of ISI on P3 latencies, P3 amplitude, and reaction time at $\mathrm{Pz}$ (Table 3) using oneway repeated measure ANOVA in PD-H patients, PD-NH patients, and controls. P3 latency was significantly prolonged at $5000 \mathrm{~ms}$ ISI compared to $1600 \mathrm{~ms}$ ISI in PD-NH patients and control (control, $F=19.289, p=0.003$; PD-NH, $F=5.391, p=0.04)$, while $\mathrm{PD}-\mathrm{H}$ patients did not show significant difference $(F=0.025, p=0.879)$. P3 amplitude showed unremarkable difference in three groups 
TABLE 2: Comparison of frontal test battery in PD patients and controls.

\begin{tabular}{|c|c|c|c|c|}
\hline $\begin{array}{l}\text { Demographic data } \\
\text { mean } \pm \text { SD }\end{array}$ & $\begin{array}{l}\text { PD-H } \\
n=12\end{array}$ & $\begin{array}{l}\mathrm{PD}-\mathrm{NH} \\
n=23\end{array}$ & $\begin{array}{c}\text { Controls } \\
n=18\end{array}$ & $p$ \\
\hline \multicolumn{5}{|l|}{ Attention } \\
\hline Stroop Test (errors) & $7.92 \pm 9.09$ & $7.33 \pm 8.45$ & $3.41 \pm 5.19$ & 0.151 \\
\hline TMT-A (s) & $122.21 \pm 87.47^{\mathrm{ab}}$ & $70.84 \pm 41.47$ & $52.76 \pm 30.27$ & 0.018 \\
\hline TMT-B (s) & $241.21 \pm 121.73$ & $134.00 \pm 106.8$ & $124.76 \pm 84.81$ & 0.264 \\
\hline Digit Span & $15.64 \pm 4.24$ & $15.88 \pm 2.76$ & $17.27 \pm 4.54$ & 0.198 \\
\hline \multicolumn{5}{|l|}{ Visual-constructional ability } \\
\hline Block Design (score) & $7.21 \pm 4.16$ & $12.08 \pm 4.05$ & $11.50 \pm 3.78$ & 0.255 \\
\hline $\mathrm{R}-\mathrm{O}$-copy (score) & $25.93 \pm 10.17^{\mathrm{ab}}$ & $32.08 \pm 6.55$ & $33.47 \pm 1.91$ & $<0.001$ \\
\hline \multicolumn{5}{|l|}{ Verbal fluency } \\
\hline Word list generation & $41.00 \pm 14.81$ & $44.21 \pm 11.29$ & $46.47 \pm 8.68$ & 0.122 \\
\hline \multicolumn{5}{|l|}{ Memory } \\
\hline Wordlist learning recall (number) & $17.21 \pm 3.66$ & $19.19 \pm 3.76$ & $22.29 \pm 1.45$ & 0.301 \\
\hline R-O-recall (score) & $7.71 \pm 6.76$ & $11.96 \pm 9.19$ & $13.00 \pm 8.32$ & 0.549 \\
\hline \multicolumn{5}{|l|}{ Higher executive function } \\
\hline Similarities (score) & $9.29 \pm 6.08$ & $10.12 \pm 7.11$ & $13.53 \pm 4.58$ & 0.760 \\
\hline Five-Point Test (correct number) & $2.79 \pm 2.46$ & $4.57 \pm 2.92$ & $5.35 \pm 2.52$ & 0.485 \\
\hline WCST-category (number) & $5.21 \pm 2.12$ & $6.00 \pm 1.71$ & $6.24 \pm 2.31$ & 0.697 \\
\hline WCST-PN/total errors (\%) & $63.00 \pm 32.91^{b}$ & $72.80 \pm 31.40^{c}$ & $25.29 \pm 20.95$ & 0.027 \\
\hline \multicolumn{5}{|l|}{ Motor programming } \\
\hline Luria's Hand Sequence (score) & $1.29 \pm 1.07^{\mathrm{ab}}$ & $2.23 \pm 1.14$ & $2.12 \pm 0.68$ & 0.019 \\
\hline
\end{tabular}

PD-H: PD patients with visual hallucinations; PD-NH: PD patients without visual hallucinations; TMT: Trail Making Test; R-O Complex Figure Test: ReyOsterrieth Complex Figure Test; WCST: Wisconsin Card Sorting Test.

$p: p$ value, by one-way analysis of covariance (ANCOVA) with age, gender, and education as covariates.

Post hoc analysis with Tukey method $\left({ }^{\mathrm{a}} \mathrm{PD}-\mathrm{H}\right.$ versus $\mathrm{PD}-\mathrm{NH},{ }^{\mathrm{b}} \mathrm{PD}-\mathrm{H}$ versus controls, and ${ }^{\mathrm{c}} \mathrm{PD}-\mathrm{NH}$ versus controls).

when comparing $1600 \mathrm{~ms}$ ISI to $5000 \mathrm{~ms}$ ISI $(\mathrm{PD}-\mathrm{H}, F=$ $0.324, p=0.585$; PD-NH, $F=2.987, p=0.112$; control, $F=1.031, p=0.344)$. Reaction time was significantly prolonged at $5000 \mathrm{~ms}$ ISI compared to $1600 \mathrm{~ms}$ ISI in PD-NH and PD-H patients (PD-NH, $F=0.359, p<0.001$; PD$\mathrm{H}, F=13.059, p=0.005$ ), while there was no significant difference in controls $(F=2.831, p=0.111)$.

3.4. The Correlations of Frontal Function and ERPs in PD$H$ Patients. The domains of frontal function in PD-H patients were analyzed for their correlation with measures of N1, P2, N2, and P3 at Pz lead. Pearson's $r$ values of correlation between P3 latency, P3 amplitude, and neuropsychological scores are shown in supplementary Table 1 (in Supplementary Material available online at http://dx.doi.org/10.1155/2016/1863508). For higher executive function (Similarities, Wisconsin Card Sorting Test), attention (Trail Making Test-type B, Digit Span), visuoconstructional ability (Digit Span, Rey-Osterrieth Complex Figure copy test), verbal fluency (word list generation), and memory (Rey-Osterrieth Complex Figure recall test), there were significant correlations for P3. However, other earlier components of N1, P2, and N2 correlations with cognitive measures were not significantly evident (data not shown).

Supplementary Table 2 summarizes the odds ratio of binary logistic regression for UPDRS-on score and P3 latency in different models. Overall, the results showed that increase of UPDRS-on scores in PD patients was associated with significantly increased risk of $\mathrm{VH}$ in four different models. After adjusting age, gender, and UPDRS-on scores, model 2 disclosed that one millisecond increase of P3 latency in PD patients was in line with $6 \%(p=0.046)$ higher risk of having $\mathrm{VH}$. By contrast, model 3 showed that there was nonsignificant trend where poor performance of Trail Making Tests, R-O copy, or Luria Hand Sequence was more likely to have $\mathrm{VH}$.

\section{Discussion}

Our study showed that nondemented PD patients with VHs had worse cognitive function than those without VHs and age-matched controls. In addition to UPDRS scores, the latency of visual P3 was associated with VH after statistically adjusting the possible confounding factors and also correlated with cognitive impairment in PD patients. In accordance with previous studies using neuropsychological assessment or functional MRI [15-18, 33, 34], our finding suggests that frontal dysfunction may play a role in the development of $\mathrm{VH}$ in nondemented PD patients.

The term of P300 is composed of mainly two distinct subcomponents, $\mathrm{P} 3 \mathrm{a}$ and $\mathrm{P} 3 \mathrm{~b}$. Although the precise functional origin of P300 induced by visual stimuli is controversial, 
TABLE 3: Comparisons of latencies and amplitude at $\mathrm{Pz}$ in visual ERPs of $\mathrm{PD}-\mathrm{H}, \mathrm{PD}-\mathrm{NH}$, and controls.

\begin{tabular}{|c|c|c|c|c|}
\hline & $\begin{array}{l}\text { PD-H } \\
n=12\end{array}$ & $\begin{array}{c}\mathrm{PD}-\mathrm{NH} \\
n=23\end{array}$ & $\begin{array}{c}\text { Controls } \\
n=18\end{array}$ & $p$ \\
\hline \multicolumn{5}{|l|}{ Amplitude, uV } \\
\hline \multicolumn{5}{|l|}{ ISI $=1600 \mathrm{~ms}$} \\
\hline N1 & $-0.59 \pm 3.19$ & $-2.48 \pm 4.75$ & $-2.57 \pm 5.18$ & 0.479 \\
\hline $\mathrm{P} 2$ & $7.05 \pm 3.81$ & $10.87 \pm 6.75$ & $7.18 \pm 5.73$ & 0.164 \\
\hline $\mathrm{N} 2$ & $-0.07 \pm 5.42$ & $0.95 \pm 7.77$ & $-1.33 \pm 6.04$ & 0.388 \\
\hline P3 & $12.19 \pm 4.74$ & $14.14 \pm 9.76$ & $11.62 \pm 7.86$ & 0.934 \\
\hline \multicolumn{5}{|l|}{$\mathrm{ISI}=5000 \mathrm{~ms}$} \\
\hline N1 & $-1.44 \pm 3.42$ & $-2.02 \pm 1.81$ & $-2.26 \pm 4.25$ & 0.351 \\
\hline $\mathrm{P} 2$ & $6.40 \pm 3.40$ & $5.83 \pm 3.81$ & $6.18 \pm 4.89$ & 0.831 \\
\hline $\mathrm{N} 2$ & $-1.57 \pm 5.08$ & $-1.24 \pm 4.39$ & $-1.48 \pm 4.32$ & 0.836 \\
\hline P3 & $11.18 \pm 5.21$ & $12.38 \pm 8.33$ & $13.34 \pm 6.50$ & 0.831 \\
\hline \multicolumn{5}{|l|}{ Latency, ms } \\
\hline \multicolumn{5}{|l|}{$\mathrm{ISI}=1600 \mathrm{~ms}$} \\
\hline N1 & $140.29 \pm 13.03$ & $139.43 \pm 21.83$ & $133.73 \pm 15.34$ & 0.585 \\
\hline P2 & $183.67 \pm 15.40$ & $185.57 \pm 22.67$ & $176.60 \pm 8.09$ & 0.643 \\
\hline N2 & $277.11 \pm 33.45$ & $262.85 \pm 18.26$ & $263.92 \pm 18.67$ & 0.288 \\
\hline P3 & $396.44 \pm 28.19^{a}$ & $366.57 \pm 21.58$ & $359.89 \pm 27.89$ & 0.005 \\
\hline \multicolumn{5}{|l|}{$\mathrm{ISI}=5000 \mathrm{~ms}$} \\
\hline N1 & $152.86 \pm 22.68$ & $131.17 \pm 20.25$ & $135.63 \pm 13.19$ & 0.594 \\
\hline P2 & $215.14 \pm 26.02$ & $191.13 \pm 29.20$ & $197.11 \pm 18.28$ & 0.391 \\
\hline $\mathrm{N} 2$ & $282.75 \pm 34.45$ & $278.00 \pm 22.94$ & $265.17 \pm 22.41$ & 0.498 \\
\hline P3 & $397.38 \pm 18.78$ & $395.89 \pm 28.29^{b}$ & $404.44 \pm 39.88^{b}$ & 0.827 \\
\hline \multicolumn{5}{|l|}{$\mathrm{RT}, \mathrm{ms}$} \\
\hline ISI $=1600 \mathrm{~ms}$ & $434.39 \pm 71.49$ & $395.65 \pm 77.05$ & $382.70 \pm 52.97$ & 0.145 \\
\hline $\mathrm{ISI}=5000 \mathrm{~ms}$ & $480.81 \pm 88.60^{b}$ & $450.64 \pm 94.03^{b}$ & $421.29 \pm 121.03^{b}$ & 0.321 \\
\hline \multicolumn{5}{|l|}{ Error rate } \\
\hline ISI $=1600 \mathrm{~ms}$ & $0.03 \pm 0.02$ & $0.04 \pm 0.05$ & $0.19 \pm 0.02$ & 0.178 \\
\hline $\mathrm{ISI}=5000 \mathrm{~ms}$ & $0.03 \pm 0.04$ & $0.02 \pm 0.02$ & $0.02 \pm 0.02$ & 0.552 \\
\hline
\end{tabular}

PD-H: PD patients with visual hallucinations; PD-NH: PD patients without visual hallucinations; RT: reaction time. Values are expressed as mean \pm SD. $p: p$ value, by one-way analysis of covariance (ANCOVA) with age, gender as covariate for between-group comparison and by one-way repeated measures analysis of variance (ANOVA) for within-group comparison.

${ }^{a} p<0.05, \mathrm{PD}-\mathrm{H}$ versus control, Tukey method for post hoc analysis,

${ }^{\mathrm{b}} p<0.05$, ISI $=5000 \mathrm{~ms}$ versus $1600 \mathrm{~ms}$, by paired $t$-test.

visual P3b represents parietal cortical distribution reflecting the top-down allocation of attention resources to relevant stimuli [35-37]. As we measured our visual P3 latency as P3b, our P3 latency may reflect the top-down attribution of visual processing.

In the present study, P3 latency with ISI of $1600 \mathrm{~ms}$ in $\mathrm{PD}-\mathrm{H}$ patients was significantly longer than control and associated with $\mathrm{VH}$ after adjustment of confounding factors. As P3 latency of ERPs increases in line with cognitive decline in Lewy body dementia patients and demented PD patients with VHs $[29,32,38]$, our finding implies that visual cognitive functions are particularly impaired in nondemented PD patients with visual hallucinations. It is accepted that VHs in PD could be related to central cholinergic dysfunction in pedunculopontine nucleus $[33,39]$. On the basis of indirect pharmacological evidence, P3 ERPs in Alzheimer's disease could reflect central cholinergic function [40, 41]. Hence, a possible explanation for our findings might be that nondemented PD patients with VHs might have more dysfunction over the frontobasal cholinergic pathways. In addition, visual ERP of fixed ISI with $1600 \mathrm{~ms}$ might be an auxiliary tool to detect cognitive dysfunction in nondemented PD.

There are several theoretical models implicated in the development of VHs in PD, and integrative approach may be needed to explore sensory, attention, and cognitive deficits [42]. Functional MRI during active VHs showed desynchronization between frontal and posterior cortical areas involved in visual processing [19], while Shine et al. suggest that decreased attentional network activity and increased primary visual system connectivity with default mode network may contribute to the development of VHs [43]. Our PD-H patient also showed significant deficits in tests about attention, visuoconstructional ability, executive function, and motor programming when comparing to $\mathrm{PD}-\mathrm{NH}$ patients and 
control. However, latencies and amplitude of N1 ERP or P2 ERP, which may be more correlated with attentional network in brain, did not show significant differences between groups.

There were several limitations in our study. First, we collect PD patients from university-based hospital and the collection bias cannot be completely excluded. Secondly, visual ERP may be affected by excessive eyelid blinking related to blepharospasm, which is common in PD [44]. We did not exclude PD patients with blepharospasm in this study but the eyeball movement artifacts are excluded from the analysis. Thirdly, neuropsychological assessment may be affected by poor attention or decreased motor function in PD patients. We arranged the assessment in the morning and patients receive regular medications before the exam, but poor attention or motor fluctuation may still happen during the time-consuming tests.

\section{Conclusion}

We found that P3 ERPs measurements may be associated with visual hallucination and cognitive impairment in nondemented PD patients. Further longitudinal follow-up may be needed to confirm whether P3 ERP measurements and visual hallucinations might predict the development of dementia in PD patients

\section{Competing Interests}

The authors declare that they have no competing interests.

\section{Acknowledgments}

This study was supported by grants from Kaohsiung Medical University Hospital (KMUH97-7G30 and KMUH-IRB980169). The authors are grateful for contributions from Professor Pang-Ying Shih. Professor Pang-Ying Shih passed away on September 1, 2013.

\section{References}

[1] D. R. Williams and A. J. Lees, "Visual hallucinations in the diagnosis of idiopathic Parkinson's disease: a retrospective autopsy study," Lancet Neurology, vol. 4, no. 10, pp. 605-610, 2005.

[2] G. Levy, M.-X. Tang, E. D. Louis et al., "The association of incident dementia with mortality in PD," Neurology, vol. 59, no. 11, pp. 1708-1713, 2002.

[3] N. Ibarretxe-Bilbao, B. Ramirez-Ruiz, C. Junque et al., "Differential progression of brain atrophy in Parkinson's disease with and without visual hallucinations," Journal of Neurology, Neurosurgery \& Psychiatry, vol. 81, no. 6, pp. 650-657, 2010.

[4] G. Fénelon, C. G. Goetz, and A. Karenberg, "Hallucinations in Parkinson disease in the prelevodopa era," Neurology, vol. 66, no. 1, pp. 93-98, 2006.

[5] D. Aarsland, K. Andersen, J. P. Larsen, A. Lolk, and P. KraghSørensen, "Prevalence and characteristics of dementia in Parkinson disease: an 8-year prospective study," Archives of Neurology, vol. 60, no. 3, pp. 387-392, 2003.
[6] D. Aarsland, K. Andersen, J. P. Larsen et al., "The rate of cognitive decline in Parkinson disease," Archives of Neurology, vol. 61, no. 12, pp. 1906-1911, 2004.

[7] N. J. Diederich, C. G. Goetz, and G. T. Stebbins, "Repeated visual hallucinations in Parkinson's disease as disturbed external/internal perceptions: Focused review and a new integrative model," Movement Disorders, vol. 20, no. 2, pp. 130-140, 2005.

[8] J. Barnes, L. Boubert, J. Harris, A. Lee, and A. S. David, "Reality monitoring and visual hallucinations in Parkinson's disease," Neuropsychologia, vol. 41, no. 5, pp. 565-574, 2003.

[9] K. Smulders, M. van Nimwegen, M. Munneke, B. R. Bloem, R. P. C. Kessels, and R. A. J. Esselink, "Involvement of specific executive functions in mobility in Parkinson's disease," Parkinsonism \& Related Disorders, vol. 19, no. 1, pp. 126-128, 2013.

[10] J. Pagonabarraga and J. Kulisevsky, "Cognitive impairment and dementia in Parkinson's disease," Neurobiology of Disease, vol. 46, no. 3, pp. 590-596, 2012.

[11] Q. Wu, L. Chen, Y. Zheng et al., "Cognitive impairment is common in Parkinson's disease without dementia in the early and middle stages in a Han Chinese cohort," Parkinsonism \& Related Disorders, vol. 18, no. 2, pp. 161-165, 2012.

[12] E. Y. Uc, M. P. McDermott, K. S. Marder et al., "Incidence of and risk factors for cognitive impairment in an early parkinson disease clinical trial cohort," Neurology, vol. 73, no. 18, pp. 14691477, 2009.

[13] B. Ramirez-Ruiz, C. Junque, M.-J. Marti, F. Valldeoriola, and E. Tolosa, "Cognitive changes in Parkinson's disease patients with visual hallucinations," Dementia and Geriatric Cognitive Disorders, vol. 23, no. 5, pp. 281-288, 2007.

[14] W. Reginold, M. J. Armstrong, S. Duff-Canning et al., “The pill questionnaire in a nondemented Parkinson's disease population," Movement Disorders, vol. 27, no. 10, pp. 1308-1311, 2012.

[15] B. Ramírez-Ruiz, C. Junqué, M.-J. Martí, F. Valldeoriola, and E. Toloso, "Neuropsychological deficits in Parkinson's disease patients with visual hallucinations," Movement Disorders, vol. 21, no. 9, pp. 1483-1487, 2006.

[16] G. Gibson, P. G. Mottram, D. J. Burn et al., "Frequency, prevalence, incidence and risk factors associated with visual hallucinations in a sample of patients with Parkinson's disease: a longitudinal 4-year study," International Journal of Geriatric Psychiatry, vol. 28, no. 6, pp. 626-631, 2013.

[17] D. Grossi, L. Trojano, M. T. Pellecchia, M. Amboni, N. A. Fragassi, and P. Barone, "Frontal dysfunction contributes to the genesis of hallucination in non-demented Parkinsonian patients," International Journal of Geriatric Psychiatry, vol. 20, no. 7, pp. 668-673, 2005.

[18] G. Llebaria, J. Pagonabarraga, M. Martínez-Corral et al., "Neuropsychological correlates of mild to severe hallucinations in Parkinson's disease," Movement Disorders, vol. 25, no. 16, pp. 2785-2791, 2010.

[19] C. G. Goetz, C. L. Vaughan, J. G. Goldman, and G. T. Stebbins, "I finally see what you see: Parkinson's disease visual hallucinations captured with functional neuroimaging," Movement Disorders, vol. 29, no. 1, pp. 115-117, 2014.

[20] T. Yamasaki, S. Horie, H. Muranaka, Y. Kaseda, Y. Mimori, and S. Tobimatsu, "Relevance of in vivo neurophysiological biomarkers for mild cognitive impairment and Alzheimer's disease," Journal of Alzheimer's Disease, vol. 31, supplement 3, pp. S137-S154, 2012. 
[21] C.-L. Lai, R.-T. Lin, L.-M. Liou, Y.-H. Yang, and C.-K. Liu, “The role of cognitive event-related potentials in executive dysfunction," Kaohsiung Journal of Medical Sciences, vol. 29, no. 12, pp. 680-686, 2013.

[22] C.-L. Lai, R.-T. Lin, L.-M. Liou, and C.-K. Liu, "The role of event-related potentials in cognitive decline in Alzheimer's disease," Clinical Neurophysiology, vol. 121, no. 2, pp. 194-199, 2010.

[23] J. Polich, J. E. Alexander, L. O. Bauer et al., "P300 topography of Amplitude/Latency correlations," Brain Topography, vol. 9, no. 4, pp. 275-282, 1997.

[24] J. Polich and T. Bondurant, "P300 sequence effects, probability, and interstimulus interval," Physiology \& Behavior, vol. 61, no. 6, pp. 843-849, 1997.

[25] H. Wang, Y. Wang, D. Wang, L. Cui, S. Tian, and Y. Zhang, "Cognitive impairment in Parkinson's disease revealed by eventrelated potential N270," Journal of the Neurological Sciences, vol. 194, no. 1, pp. 49-53, 2002.

[26] S. Prabhakar, P. Syal, and T. Srivastava, "P300 in newly diagnosed non-dementing Parkinson's disease: effect of dopaminergic drugs," Neurology India, vol. 48, no. 3, pp. 239-242, 2000.

[27] H. Tanaka, T. Koenig, R. D. Pascual-Marqui, K. Hirata, K. Kochi, and D. Lehmann, "Event-related potential and EEG measures in Parkinson's disease without and with dementia," Dementia and Geriatric Cognitive Disorders, vol. 11, no. 1, pp. 39-45, 2000.

[28] A. Aotsuka, S. J. Weate, M. E. Drake Jr., and G. W. Paulson, "Event-related potentials in Parkinson's disease," Electromyography and Clinical Neurophysiology, vol. 36, no. 4, pp. 215-220, 1996.

[29] K. Toda, H. Tachibana, M. Sugita, and K. Konishi, "P300 and reaction time in Parkinson's disease," Journal of Geriatric Psychiatry and Neurology, vol. 6, no. 3, pp. 131-136, 1993.

[30] D. O’Mahony, M. Rowan, J. Feely, D. O’Neill, J. B. Walsh, and D. Coakley, "Parkinson's dementia and Alzheimer's dementia: an evoked potential comparison," Gerontology, vol. 39, no. 4, pp. 228-240, 1993.

[31] S. Pang, J. C. Borod, A. Hernandez et al., "The auditory P300 correlates with specific cognitive deficits in Parkinson's disease," Journal of Neural Transmission-Parkinson's Disease and Dementia Section, vol. 2, no. 4, pp. 249-264, 1990.

[32] A. Kurita, M. Murakami, S. Takagi, M. Matsushima, and M. Suzuki, "Visual hallucinations and altered visual information processing in Parkinson disease and dementia with lewy bodies," Movement Disorders, vol. 25, no. 2, pp. 167-171, 2010.

[33] F. Manganelli, C. Vitale, G. Santangelo et al., "Functional involvement of central cholinergic circuits and visual hallucinations in Parkinson's disease," Brain, vol. 132, no. 9, pp. 23502355, 2009.

[34] C. Sanchez-Castaneda, R. Rene, B. Ramirez-Ruiz et al., "Frontal and associative visual areas related to visual hallucinations in dementia with lewy bodies and Parkinson's disease with dementia," Movement Disorders, vol. 25, no. 5, pp. 615-622, 2010.

[35] H. H. Fernandez and K. L. Lapane, "Predictors of mortality among nursing home residents with a diagnosis of Parkinson's disease," Medical Science Monitor, vol. 8, no. 4, pp. CR241CR246, 2002.

[36] H. H. Fernandez, K. L. Lapane, B. R. Ott, and J. H. Friedman, "Gender differences in the frequency and treatment of behavior problems in Parkinson's disease. SAGE Study Group. Systematic Assessment and Geriatric drug use via Epidemiology," Movement Disorders, vol. 15, no. 3, pp. 490-496, 2000.
[37] V. T. Nasman and P. J. Dorio, "Reduced P3b category response in prefrontal patients," International Journal of Psychophysiology, vol. 14, no. 1, pp. 61-74, 1993.

[38] I. Kimura, A. Ohnuma, H. Seki, S.-I. Saso, and K. Kogure, "Cognitive impairment in Parkinson's disease assessed by visuomotor performance system and P300 potential," The Tohoku Journal of Experimental Medicine, vol. 161, pp. 155-165, 1990.

[39] J. Janzen, D. Van'T Ent, A. W. Lemstra, H. W. Berendse, F. Barkhof, and E. M. J. Foncke, "The pedunculopontine nucleus is related to visual hallucinations in Parkinson's disease: preliminary results of a voxel-based morphometry study," Journal of Neurology, vol. 259, no. 1, pp. 147-154, 2012.

[40] A. Thomas, D. Iacono, L. Bonanni, G. D’Andreamatteo, and M. Onofrj, "Donepezil, rivastigmine, and vitamin E in Alzheimer disease: a combined P300 event-related potentials/neuropsychologic evaluation over 6 months," Clinical Neuropharmacology, vol. 24, no. 1, pp. 31-42, 2001.

[41] M. Onofrj, A. Thomas, A. L. Luciano et al., "Donepezil versus vitamin E in Alzheimer's Disease part 2: mild versus moderatesevere Alzheimer's Disease," Clinical Neuropharmacology, vol. 25, no. 4, pp. 207-215, 2002.

[42] A. J. Muller, J. M. Shine, G. M. Halliday, and S. J. G. Lewis, "Visual hallucinations in Parkinson's disease: theoretical models," Movement Disorders, vol. 29, no. 13, pp. 1591-1598, 2014.

[43] J. M. Shine, C. O'Callaghan, G. M. Halliday, and S. J. G. Lewis, "Tricks of the mind: visual hallucinations as disorders of attention," Progress in Neurobiology, vol. 116, pp. 58-65, 2014.

[44] A.-Q. Rana, A. Kabir, O. Dogu, A. Patel, and S. Khondker, "Prevalence of blepharospasm and apraxia of eyelid opening in patients with parkinsonism, cervical dystonia and essential tremor," European Neurology, vol. 68, no. 5, pp. 318-321, 2012. 


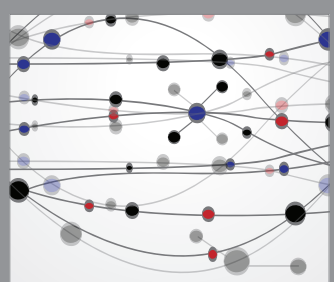

The Scientific World Journal
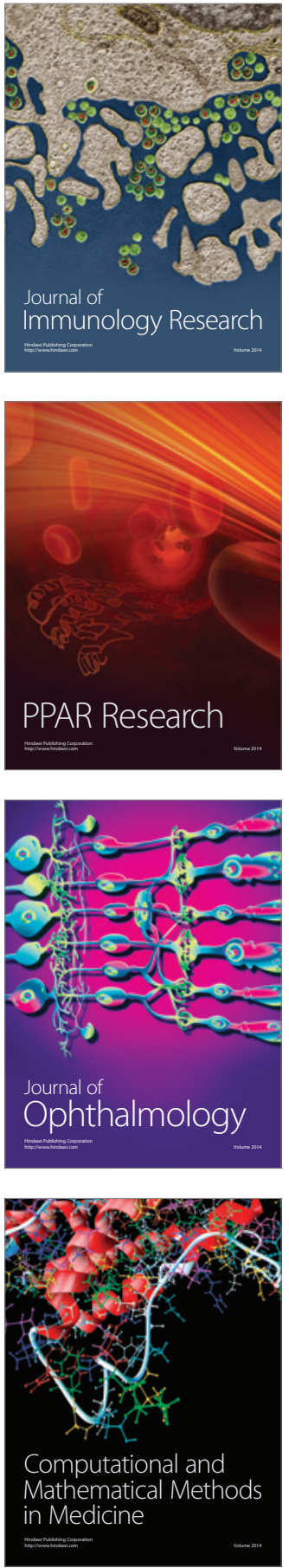

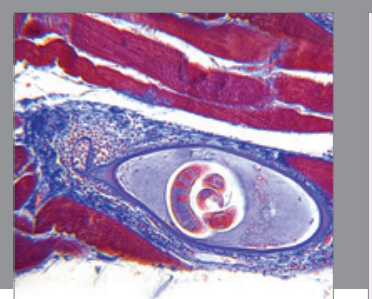

Gastroenterology Research and Practice

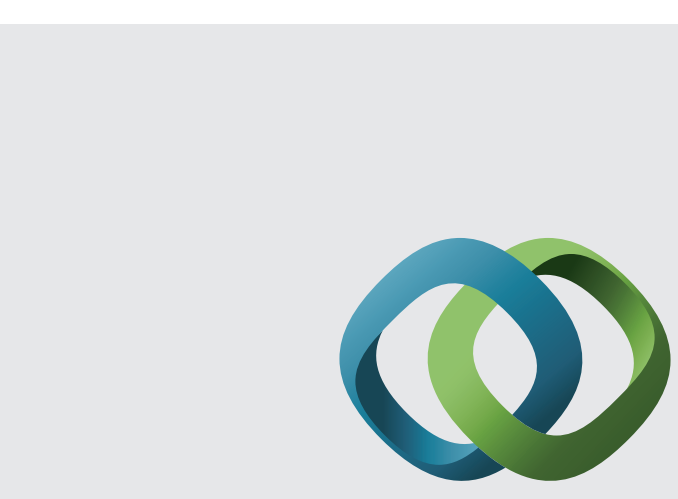

\section{Hindawi}

Submit your manuscripts at

http://www.hindawi.com
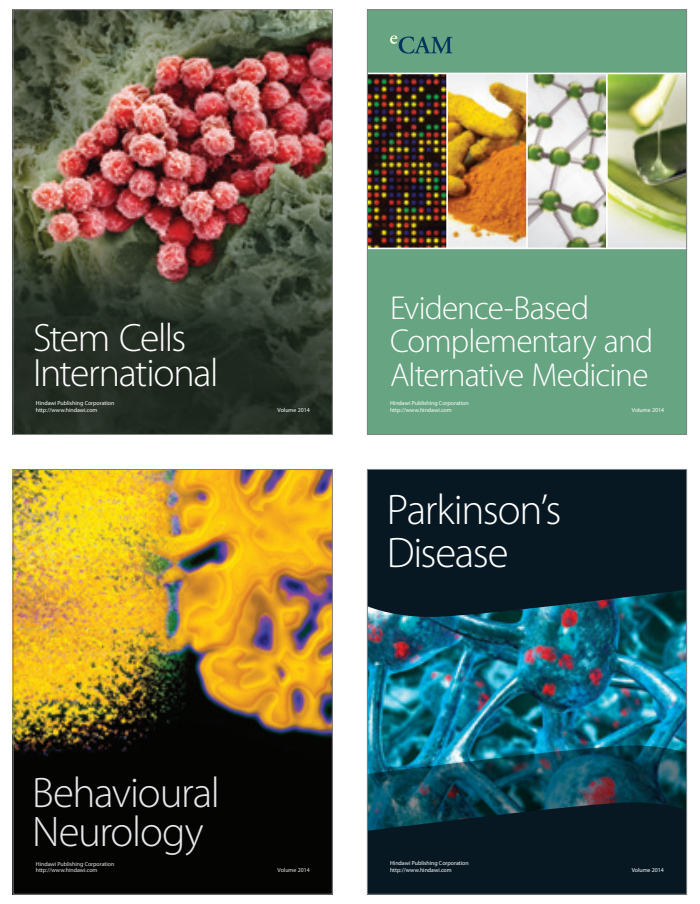
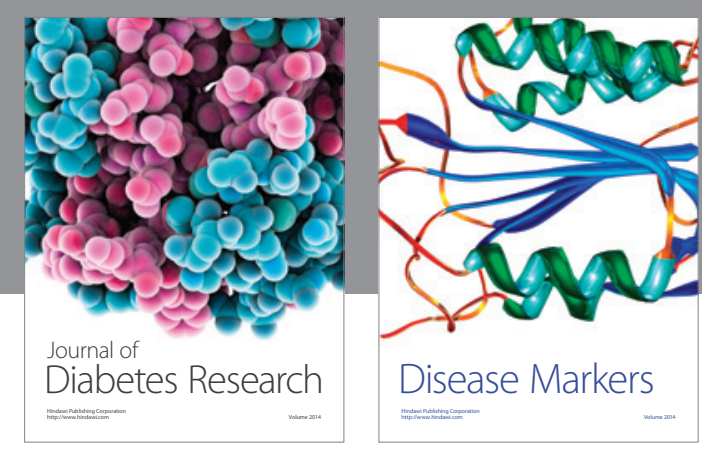

Disease Markers
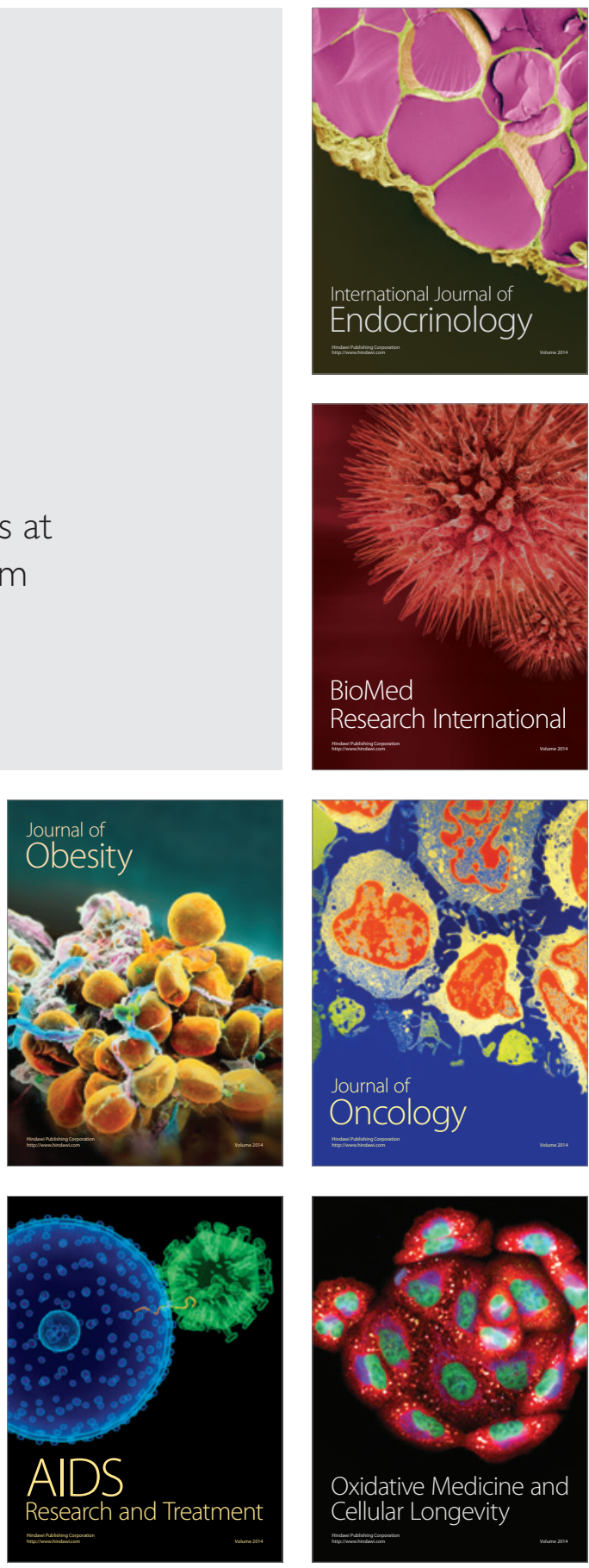